\title{
Review of Monitoring and Warning Methods for External Damage Protection of Overhead Transmission Lines
}

\author{
Yongling $\mathrm{Lu}^{1, a}$, Chengbo $\mathrm{Hu}^{2, \mathrm{~b}}$, Jiayuan $\mathrm{Xu}^{3, \mathrm{c}}$,Song $\mathrm{GaO}^{4, \mathrm{~d}}$,Fengbo $\mathrm{TaO}^{5, \mathrm{e}}$ and \\ Zhicheng Zhou ${ }^{6, f}$
}

1,2,3,4,5,6 State Grid Jiangsu Electric Power Company Research Institute, 211103, China

a15105182955@163.com, b15105168989@163.com,c15251766245@163.com, dhvgaosong@qq.com, e hvtaofb@163.com, ${ }^{f} 85752639 @ q q . c o m$

Keywords: Overhead Transmission Lines; External Damage; Monitoring and Warning

Abstract. With the rapid development of economy and power grid, contradictions continue to increase between overhead transmission lines protection and construction, more and more fault trips occur caused by external damage, monitoring and warning methods for external damage become very important to guarantee the safety of power grid. Based on extensive investigations about monitoring manufacturers, research institutions and pilot applications, this paper describes two main monitoring principle in detail, namely intelligent video monitoring and laser monitoring. The composition of each monitoring system and advantages and disadvantages are pointed out in a technical sense. Finally, it introduces the application and popularization of monitoring technology for external damage protection and puts forward reasonable suggestions for the future application.

\section{Introduction}

With the rapid development of economy and power grid, the number of power overhead lines has increased dramatically. External damage has been the major cause of fault trips [1,2]. Taking statistics of faults in 10kV and above overhead lines of 2013 Jiangsu Power Grid as an example, over the year, 4945 overhead lines were damaged, among which 1912 were caused by external factors such as vehicles, construction and floating objects, accounting for 39\% of the total number of breakdowns. Fig. 1 shows the proportion of external damage faults per month. External damage constantly causes line outage, which causes great distress on the security and stable operation of power grid [3]. Anti-external force damage is a long-term, arduous and complex work. Nowadays, construction projects are more and more frequently taken place around the overhead power line corridors [4,5], artificial site inspection is inefficient, it is urgent to expand application of monitoring technology for external damage on the grid lines.

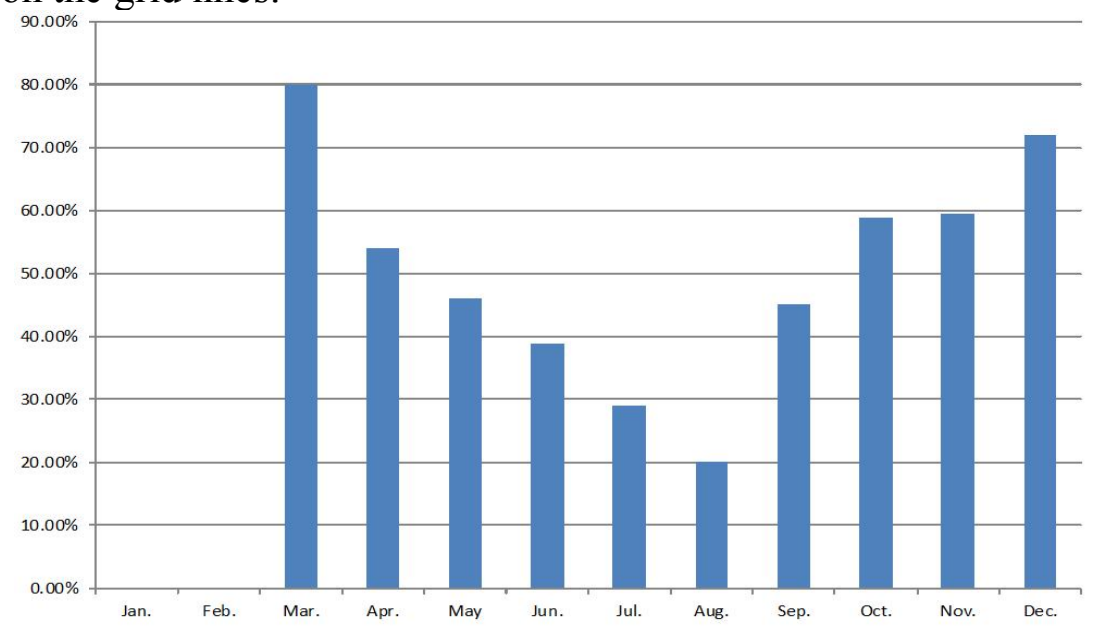

Fig.1 Proportion of external damage faults in $10 \mathrm{kV}$ and above overhead lines

At present, there are a number of agencies study the devices and monitoring system for the anti-external force damage of transmission line. Among which, Xi'an JinYuan Automation Technology Co., Shenzhen Telecom Science Technology Co., Wuhan Nari Electric Co., State Grid Fidelity, Dongfang Electronics Co. and many other companies have got whole set of technique and 
engineering practice. Their products mainly include the warning system for anti-external force damage based on intelligent video monitoring and laser monitoring.

\section{The Existing Warning Technologies of Anti-external Force Damage}

The Warning Technology for Anti-external Force Damage based on intelligent video monitoring. Based on the transmission line video monitoring technology, this technique integrates image processing techniques, reduced power consumption control technology, Wi-fi communication technology and $3 \mathrm{G}$ wireless communication technology[6,7] that especially designed for applications of transmission line. The early warning system adopts the distributed intelligence system structure to process real time video images to implement continuous video monitoring, such as motion detection and target recognition and tracking. The monitoring center will receive normal heartbeat packets until unusual on-site situation is detected. Then the front end compresses the video stream immediately to the intelligent back end that located in the monitoring center, the back end will recognize the complex behavior of the received live video by using advanced video recognition algorithm. When an unusual on-site situation is detected, an emergency warning alarm processing scheme will be started immediately. The system sketch map is shown in Fig.2.

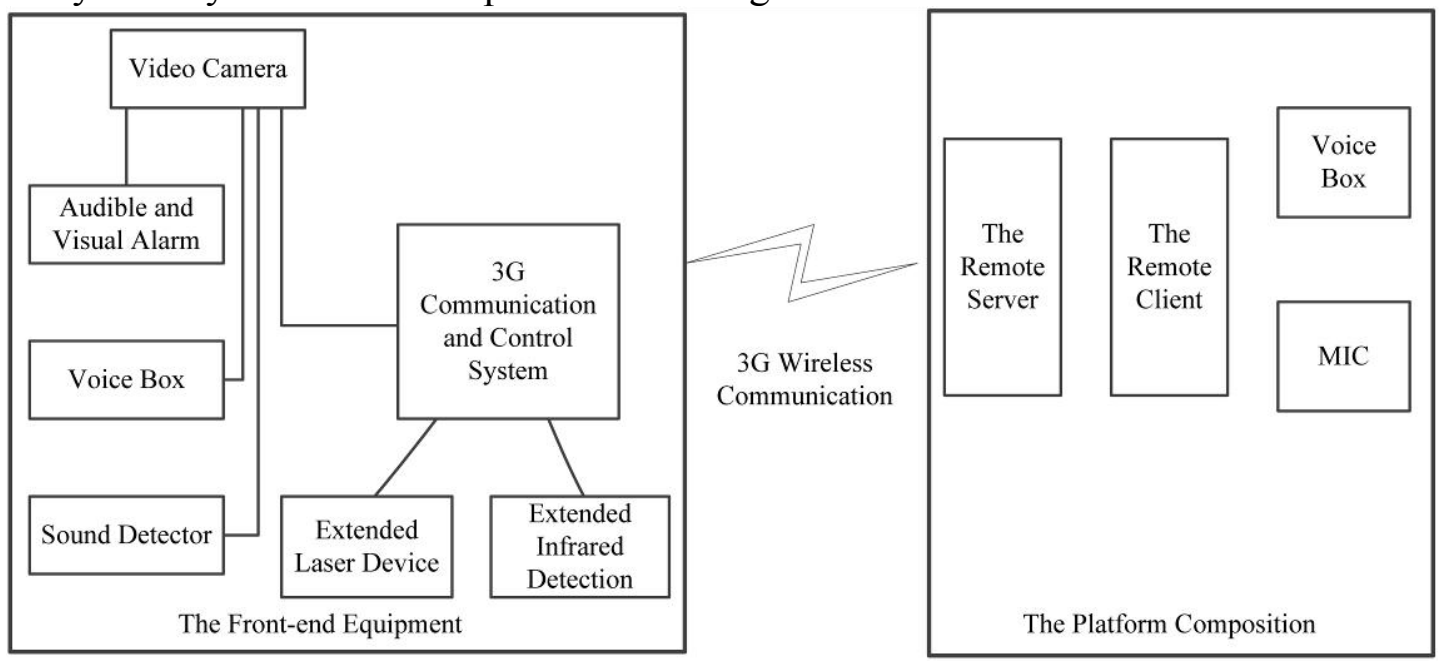

Fig.2 Composition of intelligent video monitoring system

Currently most products are based on intelligent video monitoring technology. The major producers are Xi'an JinYuan Automation Technology Co., Shenzhen Telecom Science Technology Co., Nanjing Titanium Power Technology Co., State Grid Fidelity, Dongfang Electronics Co., etc.

The Warning Technology for Anti-external Force Damage based on laser monitoring. High-voltage lines generate a tiny electric field through the air and the ground. The electric field is usually in a constant state, once a large object interrupted (especially tower crane, crane and other mechanical objects) will cause the variation of field intensity. The closer the external damage from the wire, the greater the magnitude of the electric field intensity changes. Then the distance between the object and the high-voltage line can be calculated by the variation of field intensity detected by the laser.

The principle of the early warning system based on laser monitoring is to induce the change of the electric field intensity by the physical properties of the laser, such as "Cat's eye" effect. The distance between the object and the wire can be calculated through association algorithm. Once the distance is over the predefined levels of safe distance, the system will alarm through on-site alarm horn, remote alarm or remote alarm call to stop the damage on site, or the monitor center deal with it in the background. The system can also monitor the tree condition by analyzing the relative position. According to the sensor detection, the tower tilt and clash are alarmed. The system sketch map is shown in Fig.3. 


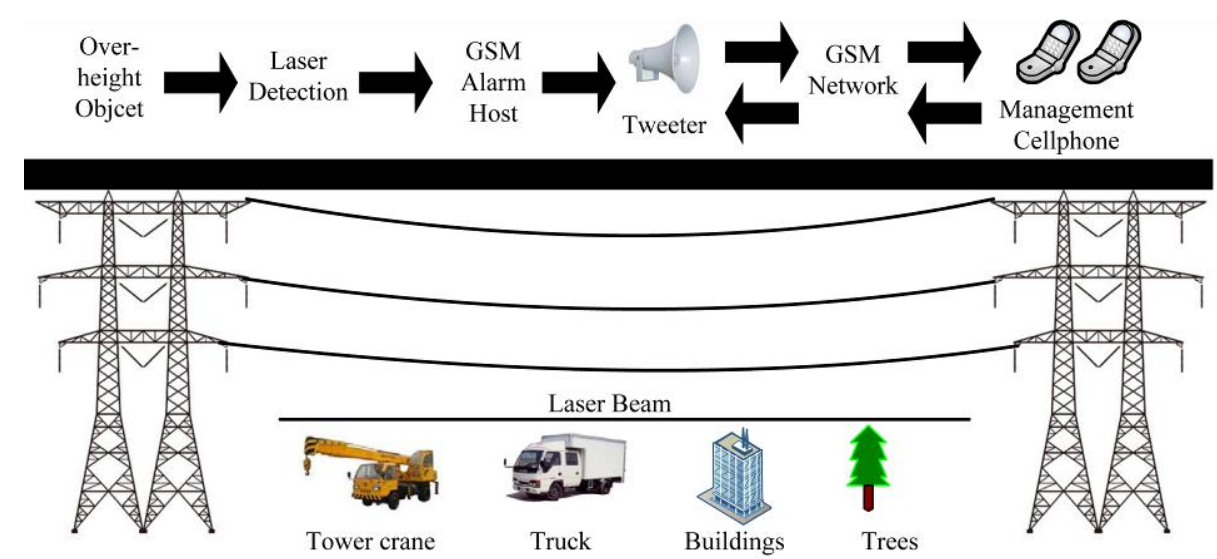

Fig.3 Laser monitoring system working principle

The major producers are Changsha Tianxi Science and Technology Development Co., Wuhan Nari Electric Co., etc.

\section{Analysis on the Structure and Performance of Anti-external Force Damage Monitoring System}

Structure of Early Warning System for Anti-external Force Damage. The early warning system for anti-external force damage of the transmission lines is mainly composed of image acquisition unit, slave monitors and background early warning software system [8,9], as shown in Fig.4.

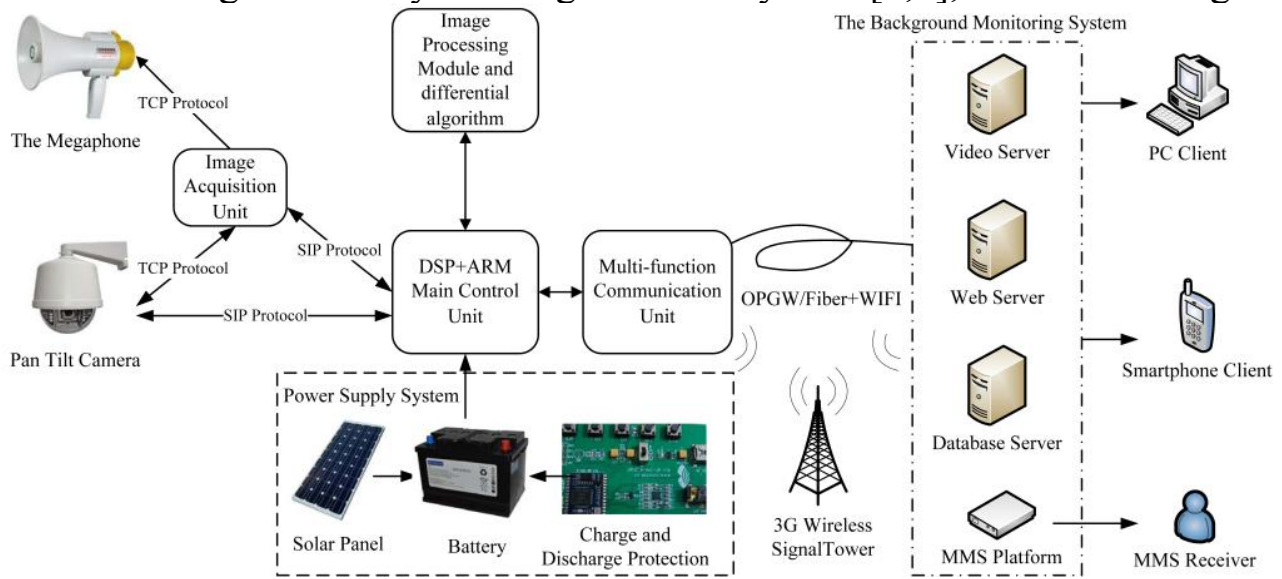

Fig.4 Structure of the monitoring and warning system

1) The Image Acquisition Unit

The image acquisition unit includes software and hardware that used to collect, transfer and control information on the site, such as video, audio, and warning and other status information. The camera is a visible light camera. The laser monitoring early warning system uses laser probe and GSM to control the hosts to realize timing controllable monitoring. The video signal captured by the video processing unit will be coding and analyzing and then sent to the monitoring center or the intelligent monitoring server through $\mathrm{CDMA} / 3 \mathrm{G} / \mathrm{OPGW}$ network. Then the diagnosis information and the abnormal image will be sent to the mobile terminal in the form of short message or multimedia message. The mobile terminal receiver can be freely set by the user to be able to achieve auto-tracking and local broadcast alerts.

2) The Slave Monitors

The slave monitor is used to interact with the intelligent monitoring server to control the power supply of the image acquisition unit. Also, with the function of data collection, storage, management and remote communication, the salve monitor can receive, analyze and send the data from the image acquisition unit to the monitoring server.

3) The Background Early Warning Software System

The background early warning software system is the core of the early warning system for anti-external force damage of the transmission lines. It is responsible for setting and managing of the intelligent analysis rules of the front-end devices. The system adopts $\mathrm{B} / \mathrm{S}$ architecture, it integrates 
with high capacity and high-speed database system, WEB release management system and MMS (multimedia message) alarm system, which is capable of displaying video, image, alarm and status information, and realizing the multi-parameter early warning.

The Performance Analysis of the Early Warning System for Anti-external Force Damage. The warning system for anti-external force damage based on intelligent video monitoring can automatic and manual remote control zoom, focus, azimuth adjustment and preset position settings. The number of pixels in the system is more than $752(\mathrm{H})$ X 582 (V) (PAL), the horizontal rotation angle can reach $0^{\circ}-355^{\circ}$, the elevation angle can reach $0^{\circ}-90^{\circ}$, the intelligent alarm delay is less than $2 \mathrm{~s}$. The monitor device can preset and remote control the camera's focal length, aperture, azimuth, and set and adjust the PTZ preset. Also, the system can be applied to both direct current and alternating current. However, the system can only detect a distance of 100 meters, and is greatly affected by the non-negligible natural weather factors (such as rain and fog, haze, fog and haze). The large number of electrical components of the system consumes a lot of energy. Without the solar power system or intermittent work mode, it will affect the overall power supply of the equipment.

The warning system for anti-external force damage based on laser monitoring can automatically calculate the distance between the object and the high-voltage line. The warning distance of the system is alterable from 0 to 800 meters, the alarm time can reach $2 \mathrm{~s} \pm 1 \mathrm{~s}$, the response time is $5 \mathrm{~ms}-500 \mathrm{~ms}$ and the distance precision is $0.5 \mathrm{~m}$. According to the safety distance parameter set for different levels of lines, the system can determine the risk gradation and achieve multi-level alarm. The system can monitor long-term point and temporary point of the high-voltage line from a long distance, and provides linkage interface with the video monitor. The whole system is light-weight, less-attachment and easy to install and maintain. The system adopts the advanced micro-detection chip to achieve low energy consumption and sufficient laser emission to ensure the effective use of the equipment. However, the change of the magnitude of the electric field intensity caused by the object adjoin to the DC transmission line is not obvious, thus the distance between the object and the high-voltage line cannot be determined by the laser principle. Therefore, the warning system based on laser monitoring is only applicable to the AC transmission line.

\section{The Application of the Early Warning System for Anti-external Force Damage}

The warning system for anti-external force damage based on intelligent video monitoring has been applied in Xinjiang, Zhejiang, Jiangsu, Beijing, Fujian and many other transmission lines [10,11], in which hundreds of sets has been put into operation in Beijing $220 \mathrm{kV}$ and below transmission lines, the system can effectively monitoring and early warning the solids, trees, large machinery. There have been false reports and false negative records of the 20 sets of video surveillance put into operation in 2014 in Zhengzhou, Henan and the pilot installation in Jiangsu Huai' an due to the influence of power supply and environment. In order to support the 2014 Youth Olympic Games, Nanjing Power Company installed video anti- external force damage warning system in the urban long-term construction area of the $110 \mathrm{kV}$ line tower in the second half of 2013, a total of 5 field terminals had been installed. During nearly a year of use, the system has played a certain effect.

The warning system for anti-external force damage based on laser monitoring has been applied to several 220kV and 500kV AC transmission lines in Sichuan, Liaoning, Fujian, Qinghai, Zhejiang and other regions since 2013. The system has successfully reported several times of external force damage, but the whole network is still far smaller than the intelligent video anti-external force damage warning system. Jiangsu Power Grid put into trial operation of a set separately in Nanjing, Huai'an and Suzhou in June-August 2014. The system operates normally currently, but the application effect still remains to be further investigated.

\section{Conclusions and recommendations}

In recent years, external damage has become the major reason for the outage of overhead transmission lines. The existing warning technologies of anti-external force damage are mainly based on intelligent video monitoring and laser monitoring. 
The warning system for anti-external force damage based on intelligent video monitoring can accurately detect the potential threat of external force damage, but affected by the weather factors and power supply, there will be false alarm or underreporting. From the viewpoint of operability, the system is a relatively safe anti-external force damage solution for the operation department and is not affected by the voltage type (AC or DC) of transmission line. In a crucial period, the system can be flexible to take manual monitoring methods to improve the success rate of anti-external force damage.

The technology of the warning system for anti-external force damage based on laser monitoring is still in the initial stage of application, it is still lack of quality and service experience. It is considerable to take a trial application on a line tower of complex condition (such as an important span tower). Further applications can be considered after performance investigation.

Because of the lack of statistical analysis on the effectiveness of the warning system, it is suggested to strengthen the statistical analysis of the system which has been tried or planned to try to improve the pertinence and effectiveness of the warning system.

\section{Acknowledgements}

This work was financially supported by the Natural Science Foundation of China (Grants No.51407026).

\section{References}

[1] Xiao An-nan, Geng Ke-shan, Liu Wu.Power Line External Damage Risk Characteristics and Countermeasures[J].JournalOfAnhui Electrical Engineering Professional Technique College,2015,20(3):26-30.

[2] Pang Hong-mei,Fan Gao-hang, Li lin. Reasons and Countermeasures for External Damage of Transmission lines [J]. Electric Safety Technology,2003,5(4): 45-46.

[3] Chen De-jun,Huang Hao-jun,Guo Hui. Characteristics and Preventive Measures of Seasonal Inspection of Overhead Transmission Lines [J]. China Science and Technology Information, 2012(8): 76-76

[4] Yang Dong, Wang Jian-cheng, Zhan Xu-liang,etal. Analysis and Countermeasures Discussion of Transmission Line Corridors Construction Broken Governance[J].Anhui Electric Power,2014,31:21-23.

[5] Zheng Yong, Wan Neng, Liu Rong-wei. Research on Protection of Transmission Lines [J]. Journal of Suzhou University, 2013, 28(8):70-72.

[6] Xu Jin-tong, Zhang Wei, Xu Rui. Research on External Damage Monitoring System of Transmission Lines based on Machine Vision [J]. Information \& Communications, 2015, (10): 66-67.

[7] LIU Guang-zhou, WU Xiang, REN Qi-jun. Application of Remote Visualization Monitoring System in Preventing Outsiding-Break of Transmission Line [J]. Journal of Anhui Electrical Engineering Professional Technique College, 2011,11(1):102-104.

[8] Huang Xin-bo,Sun Qin-dong, Wang Xiao-jing,etal. Image Remote-monitoring System of Transmission Lines Dangerous Items [J]. High Voltage Engineering, 2007，33（8）:192-197.

[9] Wang Guo-sheng, Zhang Guang-zhou. Detection System Design and Application for Over-Height Transmission Line [J]. Insulators and Surge Arresters, 2013 (5) : 112-118.

[10] Li Qiang-you, Zhang Wei-pan, Yang Lin-tao. Talking about Anti External Damage Work of Ttransmission Lines[J]. .Bei Jing Dianli Gaodeng Zhuanke Xuebao, 2012, 29(2): 41-41.

[11] Chen Ruizhang, Wang Lianhui, Liu Qibiao, etal [J].Analysis and Countermeasures of external damage of transmission lines, Electrical Technology, 2014(9):105-107. 